\title{
Correction to: Fractions of cardiovascular diseases and mental disorders attributable to psychosocial work factors in 31 countries in Europe
}

\author{
Isabelle Niedhammer ${ }^{1,2,3,4} \cdot$ Hélène Sultan-Taïeb ${ }^{5,6} \cdot$ Jean-François Chastang ${ }^{1,2,3} \cdot$ Greet Vermeylen $^{4}$. \\ Agnès Parent-Thirion ${ }^{4}$
}

Published online: 28 July 2020

(c) Springer-Verlag GmbH Germany, part of Springer Nature 2020

\section{Correction to: \\ Int Arch Occup Environ Health (2014) 87:403-411 \\ https://doi.org/10.1007/s00420-013-0879-4}

Whilst working on an update of our study published in 2014 (original article), we have discovered an error in the measure of effort-reward imbalance (ERI) in this study, leading to errors in Table 3 for (1) the prevalence of exposure to ERI, and (2) the fractions of cardiovascular diseases and mental disorders attributable to ERI, attributable fractions (AF) being calculated from exposure prevalence and relative risk. This error has led to overestimated exposure prevalences and AFs. However, the results have been unchanged for the differences between countries, as well as for the non-significance of AFs for cardiovascular diseases and the significance of AFs for mental disorders. The corrected results are presented in Table 3 (corrected). The corrections do not change our main conclusions.

The original article can be found online at https://doi.org/10.1007/ s00420-013-0879-4.

Isabelle Niedhammer

isabelle.niedhammer@inserm.fr

1 INSERM, U1018, CESP Centre for Research

in Epidemiology and Population Health, Epidemiology

of Occupational and Social Determinants of Health Team,

16 Avenue Paul Vaillant Couturier, 94807 Villejuif Cedex,

France

2 Univ Paris-Sud, UMRS 1018, Villejuif, France

3 Université de Versailles St-Quentin, UMRS 1018, Villejuif, France

4 European Foundation for the Improvement of Living and Working Conditions, Dublin, Ireland

5 Centre de recherche interdisciplinaire sur la biologie, la santé, la société et l'environnement (CINBIOSE), Université du Québec à Montréal (UQAM), Montréal, Canada

6 Laboratoire d'Economie et Gestion, Université de Bourgogne, Dijon, France 
Table 3 Fractions of cardiovascular diseases and mental disorders attributable to effort-reward imbalance (ERI) in Europe

\begin{tabular}{|c|c|c|c|c|c|c|c|c|c|}
\hline \multirow{3}{*}{$\begin{array}{l}\% \\
\text { Austria }\end{array}$} & \multicolumn{3}{|c|}{ Prevalence of ERI } & \multicolumn{3}{|c|}{ Cardiovascular diseases } & \multicolumn{3}{|c|}{ Mental disorders } \\
\hline & \multirow{2}{*}{$\begin{array}{l}\mathrm{Pe} \\
12.25\end{array}$} & \multicolumn{2}{|l|}{$95 \% \mathrm{CI}$} & \multirow{2}{*}{$\begin{array}{l}\mathrm{AF} \\
12.11\end{array}$} & \multicolumn{2}{|l|}{$95 \% \mathrm{CI}$} & \multirow{2}{*}{$\begin{array}{l}\mathrm{AF} \\
9.46\end{array}$} & \multicolumn{2}{|l|}{$95 \% \mathrm{CI}$} \\
\hline & & 10.11 & 14.38 & & -2.81 & 27.03 & & 4.73 & 14.20 \\
\hline Belgium & 9.52 & 8.23 & 10.82 & 9.79 & -2.57 & 22.15 & 7.53 & 3.76 & 11.30 \\
\hline Bulgaria & 9.39 & 7.85 & 10.94 & 9.67 & -2.59 & 21.93 & 7.43 & 3.65 & 11.21 \\
\hline Croatia & 8.23 & 6.24 & 10.22 & 8.61 & -2.54 & 19.77 & 6.57 & 3.02 & 10.12 \\
\hline Cyprus & 13.33 & 11.17 & 15.49 & 12.99 & -2.84 & 28.81 & 10.21 & 5.18 & 15.24 \\
\hline Czech Rep & 10.67 & 8.69 & 12.66 & 10.79 & -2.73 & 24.30 & 8.35 & 4.10 & 12.61 \\
\hline Denmark & 6.59 & 5.22 & 7.95 & 7.07 & -2.23 & 16.37 & 5.34 & 2.48 & 8.19 \\
\hline Estonia & 5.86 & 4.57 & 7.15 & 6.36 & -2.09 & 14.81 & 4.78 & 2.19 & 7.37 \\
\hline Finland & 7.90 & 6.71 & 9.10 & 8.32 & -2.39 & 19.03 & 6.34 & 3.10 & 9.58 \\
\hline France & 10.32 & 9.00 & 11.65 & 10.49 & -2.64 & 23.62 & 8.11 & 4.09 & 12.13 \\
\hline Germany & 12.91 & 11.24 & 14.59 & 12.66 & -2.79 & 28.10 & 9.93 & 5.10 & 14.75 \\
\hline Greece & 21.68 & 19.59 & 23.78 & 19.03 & -2.53 & 40.60 & 15.56 & 8.55 & 22.57 \\
\hline Hungary & 12.88 & 11.23 & 14.52 & 12.63 & -2.78 & 28.04 & 9.90 & 5.09 & 14.72 \\
\hline Ireland & 5.25 & 3.96 & 6.54 & 5.75 & -1.98 & 13.48 & 4.30 & 1.91 & 6.69 \\
\hline Italy & 10.84 & 8.91 & 12.76 & 10.93 & -2.73 & 24.59 & 8.47 & 4.18 & 12.77 \\
\hline Latvia & 4.47 & 3.36 & 5.57 & 4.96 & -1.78 & 11.70 & 3.69 & 1.62 & 5.75 \\
\hline Lithuania & 9.48 & 7.54 & 11.42 & 9.75 & -2.65 & 22.14 & 7.50 & 3.59 & 11.40 \\
\hline Luxembourg & 7.41 & 5.62 & 9.19 & 7.85 & -2.41 & 18.12 & 5.96 & 2.72 & 9.20 \\
\hline Malta & 8.56 & 6.31 & 10.81 & 8.91 & -2.62 & 20.45 & 6.82 & 3.09 & 10.55 \\
\hline Netherlands & 8.82 & 7.56 & 10.09 & 9.16 & -2.50 & 20.83 & 7.02 & 3.47 & 10.56 \\
\hline Norway & 4.88 & 3.61 & 6.16 & 5.39 & -1.90 & 12.67 & 4.02 & 1.75 & 6.28 \\
\hline Poland & 12.03 & 9.89 & 14.17 & 11.93 & -2.81 & 26.67 & 9.31 & 4.64 & 13.99 \\
\hline Portugal & 8.22 & 6.94 & 9.51 & 8.62 & -2.44 & 19.67 & 6.57 & 3.21 & 9.93 \\
\hline Romania & 16.83 & 14.39 & 19.28 & 15.67 & -2.82 & 34.16 & 12.54 & 6.58 & 18.50 \\
\hline Slovakia & 6.98 & 5.72 & 8.23 & 7.45 & -2.27 & 17.17 & 5.64 & 2.69 & 8.59 \\
\hline Slovenia & 14.02 & 11.60 & 16.43 & 13.53 & -2.86 & 29.92 & 10.68 & 5.41 & 15.94 \\
\hline Spain & 9.01 & 7.02 & 11.00 & 9.33 & -2.62 & 21.27 & 7.15 & 3.37 & 10.93 \\
\hline Sweden & 7.24 & 5.76 & 8.73 & 7.70 & -2.34 & 17.74 & 5.84 & 2.74 & 8.94 \\
\hline Switzerland & 10.33 & 8.89 & 11.78 & 10.50 & -2.65 & 23.65 & 8.12 & 4.07 & 12.16 \\
\hline Turkey & 30.27 & 27.25 & 33.29 & 24.18 & -1.72 & 50.07 & 20.40 & 11.73 & 29.08 \\
\hline UK & 8.19 & 6.90 & 9.47 & 8.58 & -2.43 & 19.60 & 6.55 & 3.20 & 9.90 \\
\hline Total & 12.28 & 11.54 & 13.01 & 12.14 & -2.71 & 27.00 & 9.49 & 4.95 & 14.02 \\
\hline$p$ value & $* * *$ & & & ns & & & $* * *$ & & \\
\hline
\end{tabular}

Bold: AF significantly different from 0 at $5 \%$

$P e$ prevalence of exposure, $A F$ attributable fraction, $n s$ non-significant

$p$ value for the comparison between countries: * $p<0.05 ; * * p<0.01$; *** $p<0.001$

\section{Reference}

Niedhammer I, Sultan-Taieb H, Chastang JF, Vermeylen G, ParentThirion A (2014) Fractions of cardiovascular diseases and mental disorders attributable to psychosocial work factors in 31 countries in Europe. Int Arch Occup Environ Health 87(4):403-411. https ://doi.org/10.1007/s00420-013-0879-4[doi]

Publisher's Note Springer Nature remains neutral with regard to jurisdictional claims in published maps and institutional affiliations. 